\title{
FRACTAL COMPLEXITY-BASED FEATURE EXTRACTION ALGORITHM OF COMMUNICATION SIGNALS
}

\author{
HUI WANG,${ }^{*}$ JINGCHAO LI,,${ }^{*} \dagger$ LILI GUO,${ }^{*}$ ZHENG DOU, ${ }^{*}$ \\ YUN LIN ${ }^{*}, \S$ and RUOLIN ZHOU \\ ${ }^{*}$ College of Information and Communication Engineering \\ Harbin Engineering University, Harbin, P. R. China \\ ${ }^{\dagger}$ College of Electronic and Information Engineering \\ Shanghai Dianji University, Shanghai, P. R. China \\ ${ }^{\ddagger}$ Department of Electrical and Computer Engineering \\ Western New England University, Springfield, MA, USA \\ §linyun@hrbeu.edu.cn
}

Received January 10, 2017

Revised April 1, 2017

Accepted April 7, 2017

Published June 22, 2017

\begin{abstract}
How to analyze and identify the characteristics of radiation sources and estimate the threat level by means of detecting, intercepting and locating has been the central issue of electronic support in the electronic warfare, and communication signal recognition is one of the key points to solve this issue. Aiming at accurately extracting the individual characteristics of the radiation source for the increasingly complex communication electromagnetic environment, a novel feature extraction algorithm for individual characteristics of the communication radiation source based on the fractal complexity of the signal is proposed. According to the complexity of the received signal and the situation of environmental noise, use the fractal dimension characteristics of different complexity to depict the subtle characteristics of the signal to establish the
\end{abstract}

\footnotetext{
${ }^{\S}$ Corresponding author.

This is an Open Access article published by World Scientific Publishing Company. It is distributed under the terms of the Creative Commons Attribution 4.0 (CC-BY) License. Further distribution of this work is permitted, provided the original work is properly cited.
} 
characteristic database, and then identify different broadcasting station by gray relation theory system. The simulation results demonstrate that the algorithm can achieve recognition rate of $94 \%$ even in the environment with SNR of $-10 \mathrm{~dB}$, and this provides an important theoretical basis for the accurate identification of the subtle features of the signal at low SNR in the field of information confrontation.

Keywords: Communication Signals; Feature Extraction; Fractal Dimension; Gray Relation Theory.

\section{INTRODUCTION}

In the future information battlefield, various communication equipment of radiation source individuals are required to implement important tasks, e.g. offering command, control, and intelligence. Nowadays, as electronic information technology develops more and more rapidly, and the modulation pattern of communication signal becomes more and more complex, the number of communication radiation source individual equipment also increases gradually, which makes the information acquisition of communication radiation source more and more difficult, $1+3$ and then brings great challenges for communication detection in the electronic confrontation field. In such a complex electromagnetic environment, it is difficult to meet the needs of modern battlefield by traditional means of communication detection, and using simple feature parameters, [4] such as modulation parameters, carrier frequency, bandwidth, symbol rate, electric level, cannot achieve the purpose of identification of individual radiation sources. Therefore, if we can effectively extract features of the radiation source individuals, different broadcasting stations in the complex electromagnetic environment can be analyzed and recognized $7 / 8$ By analyzing the effective characteristic attribute of the broadcasting station, and distinguishing the type of the broadcasting station, the type, location, threat level, interference object, and the nature of the enemy broadcasting station can be determined, and it has great application value for electronic reconnaissance, military strike and interfere with enemy signal, and meanwhile provides an important theoretical basis of command, control and so forth for military forces. Reference 9 presented a electrocardiogram (ECG) feature extraction method that consists of the morphology analysis, the fiducial point localization, and time intervals measurements by using both continuous and discrete wavelets transform (CWT and DWT, respectively).
The results were validated, and it is good method to extract signals' features under relatively high SNR environment. Reference 10 proposed two separate theoretical frameworks that can include existing multiple-parameter transforms as special cases to construct new types of transforms that is useful in signal processing and information security. A novel automatic modulation classification method based on the graph presentation of the cyclic spectrum is proposed in Ref. 11. Monte Carlo simulation results demonstrate that this proposed method can achieve much better classification accuracy than the traditional method. Reference 12 presents a methodology aimed at extracting features to obtain information that will highlight disturbances related to the field of power quality. It can classify disturbances effectively and with low computational effort. A novel method $\frac{13}{13}$ based on singular value decomposition and the main ridge slice of ambiguity function is presented for attaining a higher correct recognition rate of radar emitter signals in case of low signal-to-noise ratio. Reference 14 presented a complete framework that allows blind estimation of dynamic stream weights for audio-visual speech recognition based on coupled hidden Markov models. It significantly enhances the performance of the audio-visual automatic speech recognition system in all examined test conditions. Reference 15 proposed the method of wavelet transform combined with neural network for signal processing and feature extraction of EEG (Electroencephalogram) signal, which receive good recognition results.

Aiming at the recognition of modulation characteristics of different communication signals in the low SNR environment and the identification of subtle features of the same signal with different distribution noise, this paper proposes a new feature extraction algorithm based on multi-dimensional fractal dimension features. The main contributions of this paper are as follows: it selects the corresponding fractal feature extraction algorithm 
automatically according to the complexities of different signals first, in order to improve the recognition performance and reduce the complexity of the algorithm. And then, gray relation algorithm is used to classify and identify the feature to verify the effectiveness of the proposed algorithm.

\section{BASIC THEORY}

Fractal theory $\frac{16}{18}$ is called the geometry of the nature, which is a branch of modern mathematical theory. It is complementary to the dynamic system of chaos theory. In 1975, the American mathematician who born in France, B B Mandelbrot, first proposed the concept of fractal geometry. With the continuous development of fractal theory, it pointed out that any part of the world are possible (e.g. energy, function, information, time, structure, form, etc.) in a certain process or under certain conditions, which showed a similarity to the whole. The change in spatial dimension can be continuous, also can be discrete, which expanded the people's vision.

At present, there are many definitions and measurement methods of fractal dimension. The common one-dimensional fractal dimension is Hausdorff dimension, Higuchi dimension, Petrosian dimension

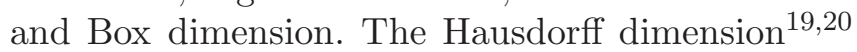
is the most basic fractal dimension. But its computational complexity is high and its computational complexity is large, which is difficult to be realized in practical communication. The Box dimension ${ }^{21}$ is relatively simple, and the fractal dimension of the extracted signal can be obtained by adjusting the size of the side length of the box. So it has been widely applied. The multi-fractal dimension 22 characterized the fractal dimension of the signal in multiple layers which based on the one-dimensional fractal dimension. And it is better to describe the subtle changes of the signal compared with the onedimensional fractal dimension. However, the complexity is increasing. Therefore, how to select the corresponding fractal dimension algorithm according to the complexity of the signal, and then accurately identify the different communication signals is the key point in today's study.

\subsection{Fractal Box Dimension}

Fractal dimension is a tool to depict complexity characteristics of object. Box dimension is one algorithm in many calculation methods which is simple and has small amount of calculation, it can well describe the complexity characteristics of the signal.
For $(X, d)$ is a metric space, $M$ is the no empty tight set family of $X$, suppose $A$ is a no empty tight set of $\mathrm{X}$, for each positive number $\varepsilon, N(A, \varepsilon)$ represent the number of the minimum box covering $A$, the side length of the box is $\varepsilon$, so $\underline{18}$

$$
N(A, \varepsilon)=\left\{M: A \subset \sum_{i=1}^{M} N\left(x_{i}, \varepsilon\right)\right\},
$$

where $x_{1}, x_{2}, \ldots, x_{M}$ are the different points of $X$, define box dimension as follows:

$$
D_{b}=\lim _{\varepsilon \rightarrow 0} \frac{\operatorname{In} N(A, \varepsilon)}{\operatorname{In}(1 / \varepsilon)} .
$$

Suppose the discrete signal $x(i)$, the highest resolution is the sampling interval $\varepsilon$, use approximate algorithm, let minimum side length of the covering box equal to signal sampling interval $\varepsilon$, calculate the grid counting $N_{k \varepsilon}$ of the box with side length $k \varepsilon$ of the discrete signal $x(i)$ in turn, so

$$
\begin{aligned}
& s_{1}= \max \left\{x_{k(i-1)+1}, x_{k(i-1)+2}, \ldots,\right. \\
&\left.x_{k(i-1)+k+1}\right\}, \\
& s_{2}= \min \left\{x_{k(i-1)+1}, x_{k(i-1)+2}, \ldots,\right. \\
&\left.x_{k(i-1)+k+1}\right\}, \\
& s(k \varepsilon)=\sum_{i=1}^{N_{0} / k}\left|s_{1}-s_{2}\right|,
\end{aligned}
$$

where $i=1,2, \ldots, N_{0} / k, N_{0}$ is the number of sampling points, and $K<N_{0}, s(k \varepsilon)$ is the scale of longitudinal coordinate of the signal, so $N_{k \varepsilon}$ can be expressed as

$$
N_{k \varepsilon}=s(k \varepsilon) / k \varepsilon+1 .
$$

Choose a section with good linear degree of fitting curve $\lg k \varepsilon \sim \lg N_{k \varepsilon}$ as the free-scale area, so

$$
\lg N_{k \varepsilon}=-d_{B} \lg k \varepsilon+b,
$$

where $k_{1} \leq k \leq k_{2}, k_{1}, k_{2}$ were the beginning and the end of the free-scale area respectively. In theory, using the least squares method to calculate the slope of the straight line, which is the fractal box dimensions to be calculated of discrete signal:

$$
D=-\frac{\left(k_{2}-k_{1}+1\right) \sum(\lg k) \cdot \lg N_{k \varepsilon}}{\left(k_{2}-k_{1}+1\right) \sum \lg ^{2} k-\left(\sum \lg k\right)^{2}} .
$$

Fractal box dimension has no absolute meaning, just has relatively value. So it is valuable to use the same method to calculate different signal's box counting dimension in practical application. 
According to the definition of fractal box dimension, after discussing its basic properties, the anti-noise performance of fractal box dimension is analyzed as follows:

Set signal sequences:

$$
y(i)=x(i)+n(i),
$$

where $i=1,2, \ldots, N, x(i)$ is the useful signal, $n(i)$ is the additive noise.

Let $N$ the number of box covering the whole plane, $N_{i}(y)$ represents the number of box covering the signal in certain signal-to-noise ratio, $N_{i}(x)$ represents the number of box covering useful signal, $N_{i}(n)$ represents the number of box covering noise, it follows:

$$
N_{i}(y)=N_{i}(x)+N_{i}(n) .
$$

So the probability of box number covering signal wave-form point in all the box can be expressed as follows:

$$
\begin{aligned}
p_{i}(y)=\frac{N_{i}(y)}{N} & =\frac{N_{i}(x)+N_{i}(n)}{N} \\
& =p_{i}(x)+\Delta_{i}(n),
\end{aligned}
$$

where $\Delta_{i}(n)=N_{i}(n) / N$, because $N$ is larger, and $N_{i}(n)$ is smaller, so

$$
p_{i}(y)=p_{i}(x)+\Delta_{i}(n)=p_{i}(x) .
$$

We can see from the formula (12) that the noise has less effect on the fractal box dimension, it has a good anti-noise property for signal identification. Therefore, it is possible to classify signal roughly using fractal box dimension characteristics.

\subsection{Higuchi Fractal Dimension}

Higuchi fractal dimension is one kind of onedimensional fractal dimension, which is defined as follows:

Suppose the time sequence is

$$
x(1), x(2), \ldots, x(N),
$$

and the reconstructed time sequence is $x_{m}^{k}$ :

$$
\begin{aligned}
x_{m}^{k}= & \{x(m), x(m+k), x(m+2 k), \ldots, \\
& \left.x\left(m+\left[\frac{N-m}{k}\right] \cdot k\right)\right\},
\end{aligned}
$$

where $N$ represents the length of the numeric sequence $x, m=1,2, \ldots, k$ represents the initial time value of the sequence, $k$ represents the time interval between two adjacent time series. Symbol $[x]$ represents the integer part of $x$, For each reconstructed time series $x_{m}^{k}$, calculate the average length of the sequence $L_{m}(k)$, then:

$$
L_{m}(k)=\frac{\begin{array}{c}
\sum_{i=1}^{[(N-m) / k]} \mid x(m+i k)-x(m \\
+(i-1) \cdot k) \mid \cdot(N-1)
\end{array}}{\left[\frac{N-m}{k}\right] \cdot k},
$$

where $N$ is the length of numeric sequence $x$, $\frac{(N-1)}{\left[\frac{N-m}{k}\right] \cdot k}$ is the normalization factor. For all, calculate the average length of the signal, where, hence, to every $k$ value, the total average length of the discrete time signal sequence can be calculated as

$$
L(k)=\sum_{m=1}^{k} L_{m}(k) .
$$

At this time, the total average length $L(k)$ of the discrete time signal sequence is proportional to the scale $k$, that is

$$
L(k) \propto k^{-D} .
$$

Take logarithm on both sides, then the following can be obtained:

$$
\ln (L(k)) \propto D \cdot \ln \left(\frac{1}{k}\right) .
$$

The least squares method is used to fit the curve $\ln \left(\frac{1}{k}\right) \cdot \ln (L(k))$, and the slope $D$ of the fitting curve is the Higuchi fractal dimension.

\subsection{Petrosian Fractal Dimension}

Singular spectrum entropy ${ }^{21}$ is a partial analysis of subtle features of signal from the perspective of singularity. Suppose the receiver simultaneously collected information from different channels with the number of $L$. Let $X_{t}=\left\{x_{t}^{1}, x_{t}^{2}, \ldots, x_{t}^{L}\right\}$ represent the received information sequence. For the signal in one of the channels, the signal after sampling is $\left\{x_{i}, i=1,2, \ldots, N\right\}$, and the sampling number is $N$.

Suppose the waveform signal consists of a series of points $\left\{y_{1}, y_{2}, \ldots, y_{N}\right\}$, binary the sequence first, and set the binary matrix $z_{i}$, then:

$$
z_{i}=\left\{\begin{array}{ll}
1, & y_{i}>\operatorname{mean}(y), \\
-1, & y_{i} \leq \operatorname{mean}(y),
\end{array} \quad i=1,2, \ldots, N,\right.
$$

where $i=1,2, \ldots, N$ represent the points' number of the signal. 
So the Petrosian fractal dimension can be defined as

$$
D=\frac{\log _{10}^{N}}{\log _{10}^{N}+\log _{10}\left(\frac{N}{N+0.4 N_{\Delta}}\right)},
$$

where $N_{\Delta}$ is the total changes' number of adjacent symbol $z_{i}$ :

$$
N_{\Delta}=\sum_{i=1}^{N-2}\left|\frac{z_{i+1}-z_{i}}{2}\right| .
$$

From the definition of Petrosian fractal dimension, it can be known that, Petrosian fractal dimension is a relatively simple definition of the fractal dimension, compared with the other fractal dimension, the calculated method is easier.

\subsection{Katz Fractal Dimension}

Suppose the waveform signal consists of a series of points $\left(x_{i}, y_{i}\right)$, the length of signal is $N$. Then Katz fractal dimension is given by

$$
D=\frac{\log (N)}{\log (N)+\log \left(\frac{d}{L}\right)},
$$

where define $L$ as the length of the waveform, and $L$ can be described as follows:

$$
L=\sum_{i=0}^{N-2} \sqrt{\left(y_{i+1}-y_{i}\right)^{2}+\left(x_{i+1}-x_{i}\right)^{2}} .
$$

Define $d$ as the maximum distance from the initial point $\left(x_{1}, y_{1}\right)$ to the other points, then the $d$ can be calculated as

$$
d=\max \left(\sqrt{\left(x_{i}-x_{1}\right)^{2}-\left(y_{i}-y_{1}\right)^{2}}\right) .
$$

\subsection{Sevcik Fractal Dimension}

Similarly, suppose the waveform signal consist of a series of points $\left(x_{i}, y_{i}\right)$, the length of the signal is $N$.

Normalize the signal first, then

$$
x_{i}^{*}=\frac{x_{i}-x_{\min }}{x_{\max }-x_{\min }}, \quad y_{i}^{*}=\frac{y_{i}-y_{\min }}{y_{\max }-y_{\min }} .
$$

So the Sevcik fractal dimension $D$ can be calculated as

$$
D=1+\frac{\ln (L)+\ln (\underline{2})}{\ln [2 \times(N-1)]},
$$

where $L$ is the length of the waveform, and $L$ can be calculated as:

$$
L=\sum_{i=0}^{N-2} \sqrt{\left(y_{i+1}^{*}-y_{i}^{*}\right)^{2}+\left(x_{i+1}^{*}-x_{i}^{*}\right)^{2}} .
$$

\subsection{Multi-Fractal Dimension}

Multi-fractal dimension is used to describe signal characteristics of different levels, and it is also used to discuss the probability distribution of parameters. It divides the research object (set its linear degree equal to 1) into $N$ small areas. Suppose the line degree of areas $i$ is $\varepsilon_{i}$, and the density distribution function $P_{i}$ of area $i$ can be described by different scaling index $\alpha_{i}$ as

$$
P_{i}=\varepsilon_{i}^{\alpha_{i}}, \quad i=1,2, \ldots, N_{i} .
$$

Non-integer $\alpha_{i}$ can commonly be called as singular index, and its value is relevant to its region.

In order to get a series of distribution characteristics of the subset, define the function $X_{q}(\varepsilon)$ as follows, which is the weighting summation of probability in each area.

$$
X_{q}(\varepsilon)=\sum_{i=1}^{N} P_{i}^{q} .
$$

Then, further define the generalized fractal dimension $D_{q}$ as

$$
\begin{aligned}
D_{q} & =\frac{1}{q-1} \lim _{\varepsilon \rightarrow 0} \frac{\ln X_{q}(\varepsilon)}{\ln \epsilon} \\
& =\frac{1}{q-1} \lim _{\varepsilon \rightarrow 0} \frac{\ln \left(\sum_{i-1}^{N}\right)}{\ln \varepsilon} .
\end{aligned}
$$

$X_{q}(\varepsilon)$ reflects the different effects of $P_{i}$. The formula shows that, when $q \gg 1$, large probability area plays the leading role in the summation formula $\sum_{i=1}^{N} P_{i}^{q}$, and at this time, $X_{q}(\varepsilon)$ and $D_{q}$ reflect the properties of big probability area. Conversely, when $q \ll 1, X_{q}(\varepsilon)$ and $D_{q}$ reflect the properties of small probability areas (sparse area). In this way, different $q$ values reflect the properties of different probability characteristic areas. Through the weighting summation processing, it divides the signal into many areas with different singular degree, and therefore the signal's internal structure is hierarchical.

When $q=0,1,2, D_{q}$ is defined as capacity dimension (box dimension) $D_{0}$, information dimension $D_{1}$ and correlation dimension $D_{2}$, respectively.

Through the phase space reconstruction of signal, then calculate the value of $P_{i}$, so different probability characteristics of the signal can be extracted, and multi-level feature results can be obtained.

In summary, six commonly used fractal dimension algorithms were defined, and the waveform features can be extracted. The calculation method of 
Higuchi fractal dimension, Petrosian fractal dimension, Katz fractal dimension and Sevcik fractal dimension is simpler to some extent. But the feature extracted is not precise, so it is suitable for rough features extraction. Compared with other fractal dimension algorithms, fractal box dimensions can extract the waveform features more precisely. So it widely applied in many fields. And the computational complexity is also increasing. Multifractal dimension can describe the characteristics of the signal from different perspective, the computational complexity also increased. It has better application for the subtle features' extraction of the same signal in different noise environments. Therefore, this paper chooses the corresponding fractal dimension feature extraction algorithm based on the prior analysis of the signal and noise complexity, and makes an optimal choice in the complexity and effectiveness of the algorithm.

\section{SYSTEM INDENTIFICATION MODEL}

Subtle feature extraction is the key link in the process of radiation source identification. The quality of the extracted features directly affects the recognition rate and the complexity of the subsequent classifier design, so the subtle feature extraction occupies a pivotal position in the communication signals' identification system. Aiming at the problem that the signals' features are difficult to be extracted in complex electromagnetic environment, this paper proposes a multi-dimensional fractal dimension based signal feature extraction algorithm, which characterizes the modulation complexity of communication signals from different geometrical scales, and then achieves the purpose of different complexity signals' recognition. According to the type and complexity of the signals, onedimensional fractal dimension can be first chosen to extract the rough features of different modulation signals. For the signals with poor separation degree, multi-fractal dimension algorithm was used to extract the detailed features of the signals. For the same signal with different noise in the low SNR environment, it is difficult to characterize the features using the simple fractal dimension. So phase space reconstruction is directly used, then calculate the multi-fractal dimension of the signals to realize the recognition of noise's subtle feature. Finally, gray relation algorithm is used to realize the accurate classification of the extracted features. So the

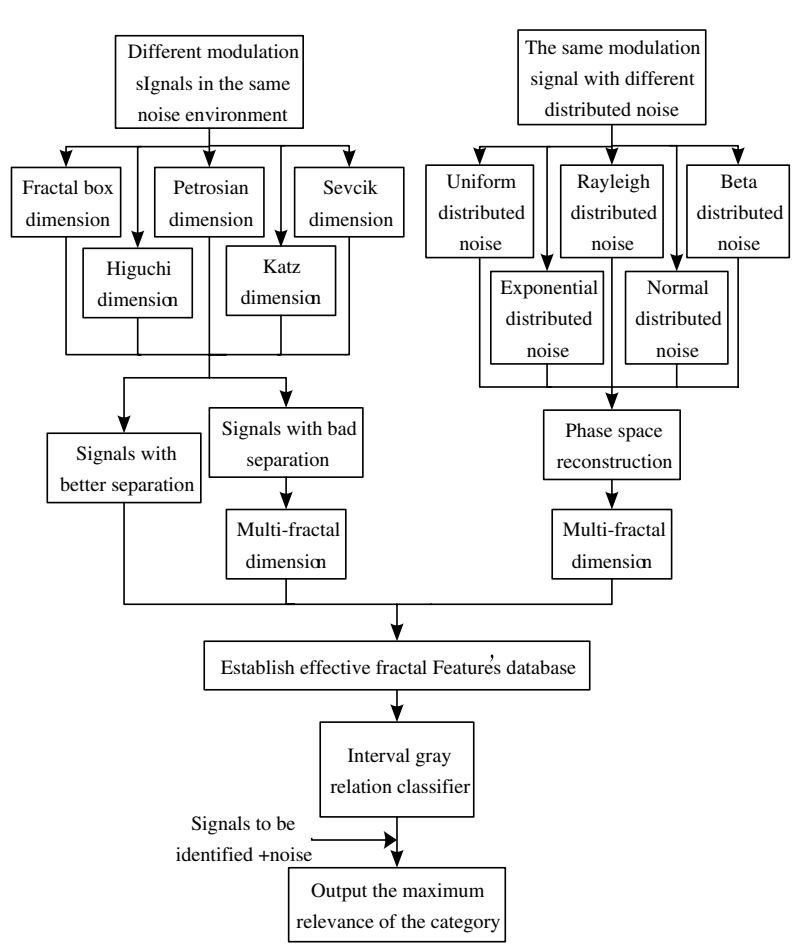

Fig. 1 Multi-fractal dimension-based recognition system model of communication signals.

effectiveness of the proposed algorithm can be verified. The flow chart of the identification system can be shown in Fig. 1.

The subtle features of individual signals can be seen as a time-varying random function. Generally speaking, for the pure internal noise, the individual nuances from different signals can be distinguished by waveforms. This is a process to extract the signals' features from global to local, macro to micro. Fractal theory is similar with it in nature, it is a general term for self-similar structures with no characteristic length but a certain meaning. It has a fine structure and a certain self-similarity in statistics, in which the fractal box dimension reflects the geometric scale of the signal sequence, and the information dimension can reflect the information of the signal sequence on the distribution density. The subtle features of the communication signals can be extracted by using the noise characteristic of the radiation source, which is mainly reflected in the amplitude, frequency, and phase of the internal noise. The characteristics of the noise include their geometric and distributed information. Therefore, it is possible to use the fractal dimension to describe the complexity of the radio signal, so it is possible to extract the subtle features of the radio station. Furthermore, using the extended multi-fractal dimension to extract the 
features of noise from different levels, and describe the waveform points from different probability, it can improve the number of differentiated noise categories and to more accurately identify the nuances of different types of radiation sources.

\section{BASIC STEPS OF PROPOSED ALGORITHM}

According to the analysis of system block diagram, the corresponding fractal dimension characteristics can be selected according to the signals' complexity and different SNR environment to extract the subtle features of the signals. Specific implementation steps can be described as follows.

\subsection{Feature Extraction of Different Modulation Signals with the Same Noise}

For different modulated signals with the same white noise, the feature is more obvious than the signals with different noise distribution. In general, it is not necessary to use multi-fractal dimension directly to extract the features which has higher complexity. Therefore, for different communication modulation signals in the relatively higher SNR environment, roughly classify the signals first and then classify the signals in detail. It has less amount of calculation. For the signals that cannot be accurately identified in low SNR, multi-fractal dimension algorithm can be used to calculate, the specific steps are as follows:

(1) Firstly, using one-dimensional fractal dimension algorithm that introduced in Secs. 2.1 2.5 to roughly extract the signals' features first.

(2) Secondly, for the signals with poor separation degree, multi-fractal dimension can be used to carry out the secondary classification, in order to realize the subtle features' extraction.

\subsection{Feature Extraction of the Same Modulation Signals with Different Noise}

For the same communication signal with different noise, the characteristics of the signal are not obvious. Therefore, it is necessary to reconstruct the phase space of the signal, and then use multi-fractal dimension to extract the subtle features. Compared with the algorithm that deal with different signals with the same noise, the complexity of this algorithm is more, but the effect of classification result is also greatly improved, the specific implementation steps are as follows:

(1) First of all, preprocess the received unknown communication signal, and discretizing the signal first:

Assuming that the received individual signal is $s$, the discrete signal sequence after preprocessing is $\{s(i)\}$, where $i=1,2, \ldots, N_{0}$ is the number of sampling points, $N_{0}$ represent the length of the signal sequence,

(2) Reconstruct the discretized signal sequence: First, define the following characteristic parameters of the preprocessed discrete communication signal sequence $\{s(i)\}, i=1,2, \ldots, N_{0}$ :

Definition: $n=\log _{2}^{N_{0}}$ represents the number of different vectors of reconstructed signals;

Definition: $t(j)=2^{j}$ represents the number of discrete signal's points in each reconstructed signal, where, $j=1,2, \ldots, n$ denotes the value of different vectors' number of reconstructed signal;

Defining the numeric sequences:

$$
T(j)=\frac{N_{0}}{t(j)}=\frac{N_{0}}{2^{j}},
$$

where $j=1,2, \ldots, n$;

The definition of reconstructed signal sequence $S(j)$ is:

$$
S(j)=s\left(T(j) *(t(j)-1)+T_{0}(j)\right),
$$

where $T_{0}(j)=[1: T(j)], j=1,2, \ldots, n$;

(3) The multi-fractal dimension of the reconstructed vector is chosen, and the fractal dimension of the signal is extracted.

Multi-fractal dimension can be regarded as a union of fractal subsets with different dimensions. Suppose the research object is divided into $M$ small areas, and the line degree of the $i$ th area is $\varepsilon_{i}$, the density distribution function of the $i$ th region is $P_{i}$, then the scaling exponent $\alpha_{i}$ of different region $i$ can be described as

$$
P_{i}=\varepsilon_{i}^{\alpha_{i}}, \quad i=1,2, \ldots, N_{i} .
$$

The non-integer $\alpha_{i}$ is called singular exponent, it represents the fractal dimension of a region. Since a signal can be divided into many different small regions, a variable $f(\alpha)$ can be composed of a series of different variables $\alpha_{i}$, and $f(\alpha)$ is the multifractal dimension of the signal. 
Define the function $X_{q}(\varepsilon)$ as the weighted sum of various regions' probability, $\varepsilon$ is the line degree of the area, $q$ is the power of density distribution function $P_{i}$, that is

$$
X_{q}(\varepsilon)=\sum_{i=1}^{N} P_{i}^{q} .
$$

The definition of generalized fractal dimension $D_{q}$ is

$$
\begin{aligned}
D_{q} & =\frac{1}{q-1} \lim _{\varepsilon \rightarrow 0} \frac{\ln X_{q}(\varepsilon)}{\ln \varepsilon} \\
& =\frac{1}{q-1} \lim _{\varepsilon \rightarrow 0} \frac{\ln \left(\sum_{i=1}^{N} P_{i}^{q}\right)}{\ln \varepsilon} .
\end{aligned}
$$

Thus, each of the reconstructed signals $S(j)$ in step (2) is summed together, $S(j)$ represents the $j$ th reconstructed signal, that is

$$
\begin{aligned}
S_{J} & =\sum S(j) \\
& =\sum s\left(T(j) *(t(j)-1)+T_{0}(j)\right) \\
& =\sum_{T_{0}(j)=1}^{T(j)} s\left(T(j) *(t(j)-1)+T_{0}(j)\right),
\end{aligned}
$$

where $J=1,2, \ldots, J_{0}, j=1,2, \ldots, n, S_{J}$ is the sum of $J$ th reconstructed signals, $J_{0}$ is the number of reconstructed signals.

And then sum the entire discrete signal sequence, the result is $S$, that is

$$
S=\sum_{i=1}^{N_{0}} s(i),
$$

where $i=1,2, \ldots, N_{0}, s(i)$ is the $i$ th sampling point value of discrete signal sequence, then the $J$ th probability measure degree $P_{J}$ can be defined as

$$
P_{J}=\frac{S_{J}}{S},
$$

where $J=1,2, \ldots, J_{0}$, take $P_{J}$ into the multifractal dimension formula to calculate $D_{q}$, then the multi-fractal dimension of the signal can be obtained.

\subsection{Realization of Gray Relational Classifier}

Gray relation theory was used to calculate the relation degree of the extracted unknown signal's multi-fractal dimension with the known modulation signal's features in the database. It is judged that the modulation type of the unknown signal is the modulation type of the signal in the database with the highest relation degree. Hence, identification of individual signals can be realized, the specific steps are as follows:

Set the $q$ value form $-q_{0}$ to $q_{0}$, then $2 q_{0}+1$ number of multi-fractal dimension can be obtained. Every $q$ value has $n=\log _{2}^{N_{0}}$ number of characteristic points. For one individual signal, the feature vector is composed of $N=\left(2 q_{0}+1\right) * n=\left(2 q_{0}+1\right) *$ $\log _{2}^{N_{0}}$ number of feature points. So multi-fractal feature sequence $F_{0}$ of unknown signal can be constructed. Gray relation theory is used to calculate the characteristic sequence of the unknown signal with the feature sequence $F_{i}$ in the database. Suppose $\gamma\left(F_{0}, F_{i}\right)$ to be the relation degree of the two sequences and there are $k$ kinds of different emitter templates, that is the types of individual signals are $i=1,2, \ldots, k$, so the constituted characteristic matrix can be expressed as

$$
\begin{aligned}
F_{0}= & \left(D_{-q_{0}}^{0}(1), D_{-q_{0}}^{0}(2), \ldots, D_{-q_{0}}^{0}(n),\right. \\
& D_{-q_{0}+1}^{0}(1), \ldots, D_{-q_{0}+1}^{0}(n), \ldots, D_{q_{0}}^{0}(1), \\
& \left.D_{q_{0}}^{0}(2), \ldots, D_{q_{0}}^{0}(n)\right) \\
F_{1}= & \left(D_{-q_{0}}^{1}(1), D_{-q_{0}}^{1}(2), \ldots, D_{-q_{0}}^{1}(n),\right. \\
& D_{-q_{0}+1}^{1}(1), \ldots, D_{-q_{0}+1}^{1}(n), \ldots, D_{q_{0}}^{1}(1), \\
& \left.D_{q_{0}}^{1}(2), \ldots, D_{q_{0}}^{1}(n)\right) \\
& \vdots \\
F_{i}= & \left(D_{-q_{0}}^{i}(1), D_{-q_{0}}^{i}(2), \ldots, D_{-q_{0}}^{i}(n),\right. \\
& D_{-q_{0}+1}^{i}(1), \ldots, D_{-q_{0}+1}^{i}(n), \ldots, D_{q_{0}}^{i}(1), \\
& \left.D_{q_{0}}^{i}(2), \ldots, D_{q_{0}}^{i}(n)\right) \\
& \vdots \\
F_{k}= & \left(D_{-q_{0}}^{k}(1), D_{-q_{0}}^{k}(2), \ldots, D_{-q_{0}}^{k}(n),\right. \\
& D_{-q_{0}+1}^{k}(1), \ldots, D_{-q_{0}+1}^{k}(n), \ldots, D_{q_{0}}^{k}(1), \\
& \left.D_{q_{0}}^{k}(2), \ldots, D_{q_{0}}^{k}(n)\right),
\end{aligned}
$$

where $i=1,2, \ldots, k$ represents the number of the modulation type.

So the calculated method of relation coefficient $\gamma\left(F_{0}, F_{i}\right)$ is defined as

$$
\begin{aligned}
& \min _{i} \min _{N}\left|F_{0}(N)-F_{i}(N)\right| \\
& \gamma\left(F_{0}, F_{i}\right)=\frac{+\rho \max _{i} \max _{N}\left|F_{0}(N)-F_{i}(N)\right|}{\left|F_{0}(N)-F_{i}(N)\right|} \\
& +\rho \max _{i} \max _{N}\left|F_{0}(N)-F_{i}(N)\right|
\end{aligned}
$$


$N=1,2, \ldots,\left(2 q_{0}+1\right) * \log _{2}^{N_{0}}$ represents $N$ th characteristic of every signal's vector, $\rho$ is the resolution coefficient, and $\rho \in(0,1)$, here it sets as 0.5 . Hence gray relation degree $\gamma_{0}\left(F_{0}, F_{i}\right)$ of unknown signal $F_{0}$ 's multi-fractal dimension with the individual template radiation source in the database is defined as

$$
\gamma_{0}\left(F_{0}, F_{i}\right)=\frac{1}{k} \sum_{i=1}^{k} \gamma\left(F_{0}, F_{i}\right) .
$$

The gray relation degree is the correlation value of the unknown signal with the signal in the known database, through the calculated results to judge the type of the unknown signals, thus to achieve the classification of individual signal.

\section{SIMULATION RESULTS AND ANALYSIS}

Experiment 1. The classification of different modulation signals under the same noise environment

Take six different modulation communication signals AM, FM, PM, ASK, FSK and PSK with the same white noise added as examples to get the simulation. In the SNR range of $-10 \mathrm{~dB}-20 \mathrm{~dB}$, the fractal box dimension, Higuchi fractal dimension, Petrosian fractal dimension, Katz fractal dimension and Sevcik fractal dimension values are calculated and the characteristic curves are drawn. The simulation results are shown in Figs. 26. While, in the figures, $x$-coordinate represents SNR, and $y$ coordinate represents different fractal dimensions.

From the simulation results in Figs. 26 6 it can be seen that one-dimensional fractal dimension is difficult to classify accurately for FM and PM signals, ASK, FSK and PSK signals. If the signals can be classified by one-dimensional fractal dimension, we will use this algorithm first due to its simple and easy calculation. If the signals are difficult to use one-dimensional fractal dimension to classify, multifractal dimension should be used although it has relatively higher calculated complexity. The multifractal dimension characteristics of the different signals under the three SNR environment of $-10 \mathrm{~dB}$, $0 \mathrm{~dB}$ and $10 \mathrm{~dB}$ are shown in Figs. 769 While, $x$-coordinate Ine reprensents $\ln \varepsilon$, and $\mathrm{y}$-coordinate Inxq represents $\ln X_{q}(\varepsilon)$ in equation (34).

Experiment 2. Classification of the same signal under different SNR environments

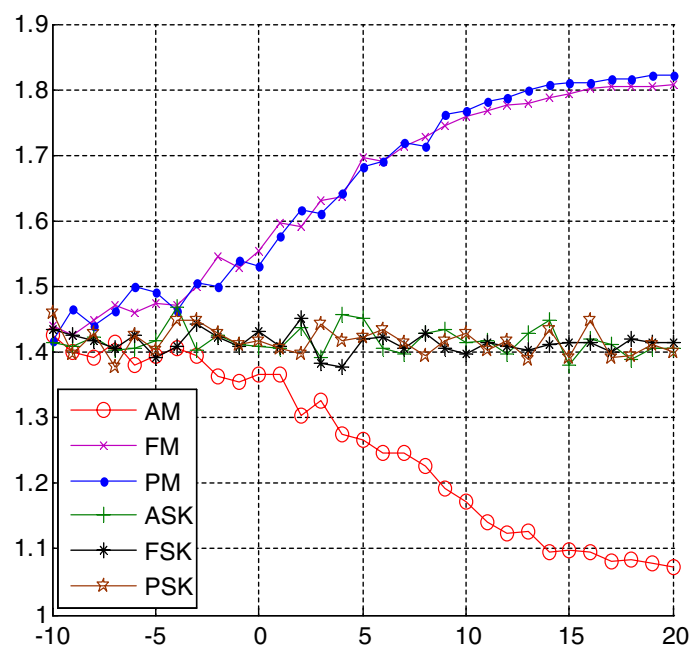

Fig. 2 Fractal box dimension-based features.

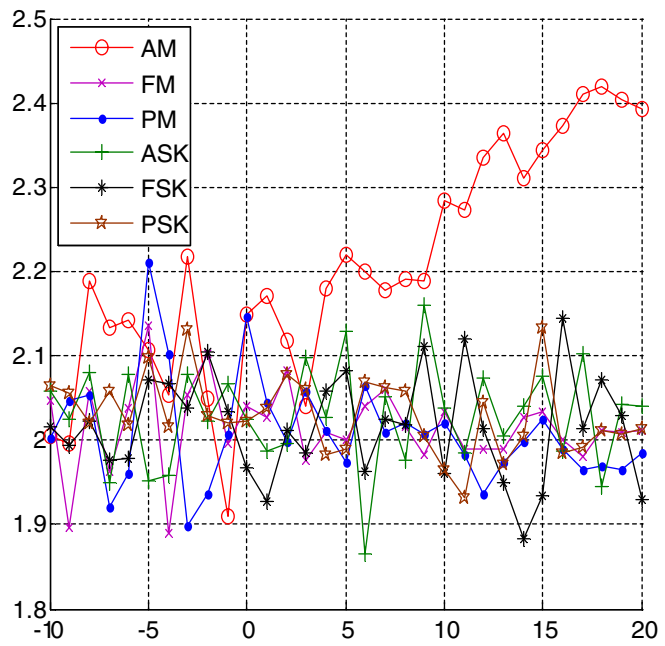

Fig. 3 Higuchi dimension-based features.

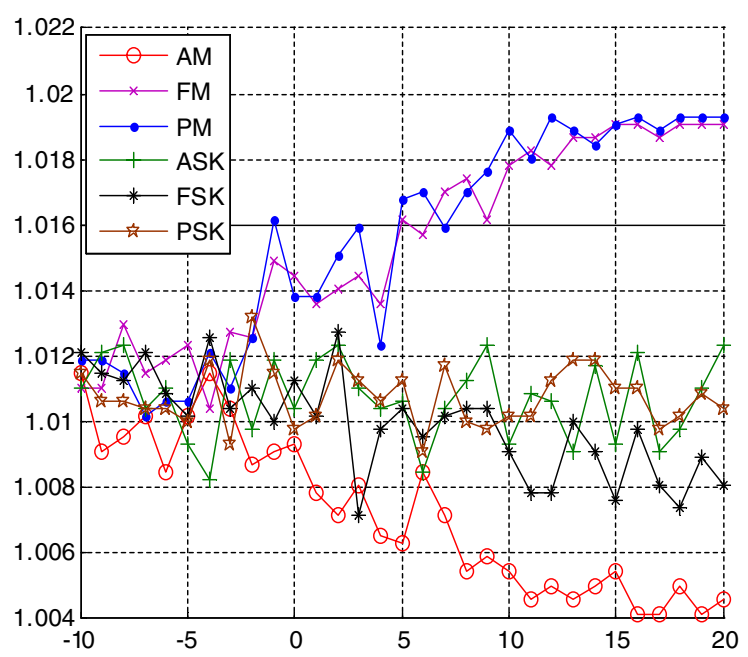

Fig. 4 Petrosian dimension-based features. 


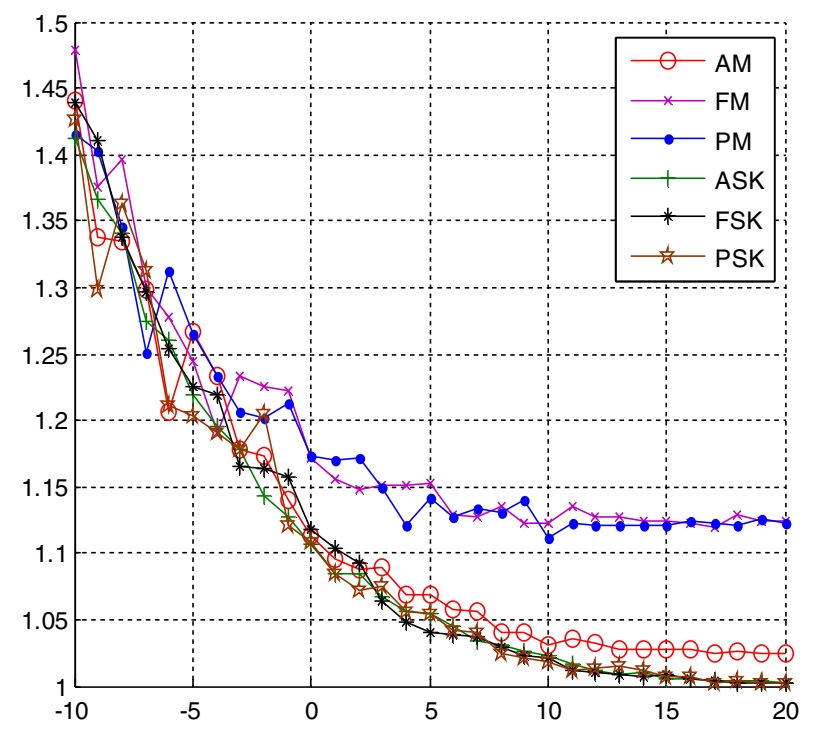

Fig. 5 Katz dimension-based features.

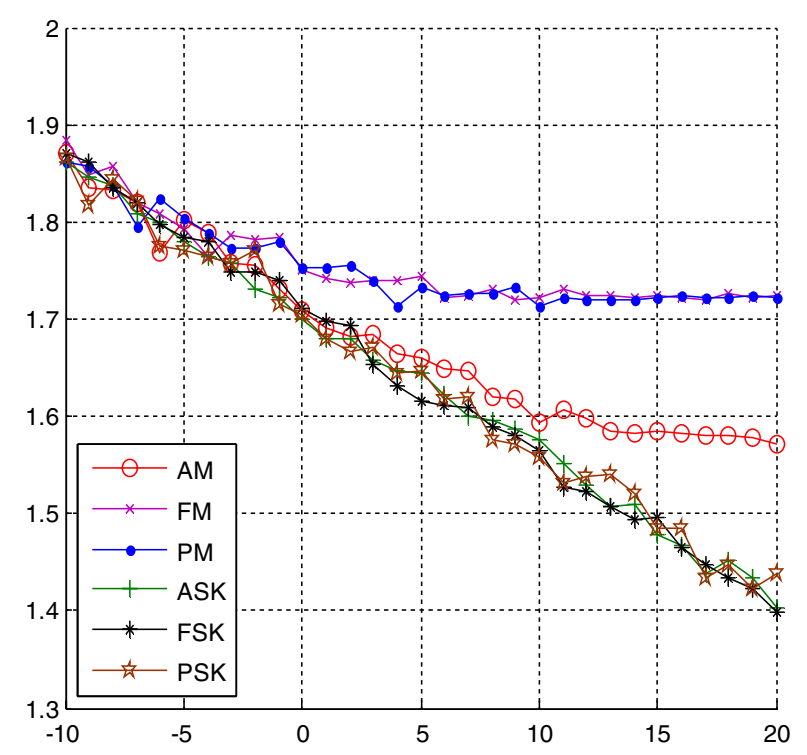

Fig. 6 Sevcik dimension-based features.

Take FSK signals with different distributions noise as examples - uniformly distributed noise, Rayleigh distributed noise, exponential distributed noise, beta distributed noise, and normal distributed noise. It is difficult to characterize the subtle features of the noise by one-dimensional fractal dimension. Therefore, phase space reconstruction is directly carried out on the signals, and then multi-fractal dimension is used to extract the noise's subtle features, and finally to extract the communication stations' "fingerprint" features. Simulation results are shown in Fig. 10. The figures on left represents the waveforms of FSK signals with different distributed noise, $x$-coordinate represents the
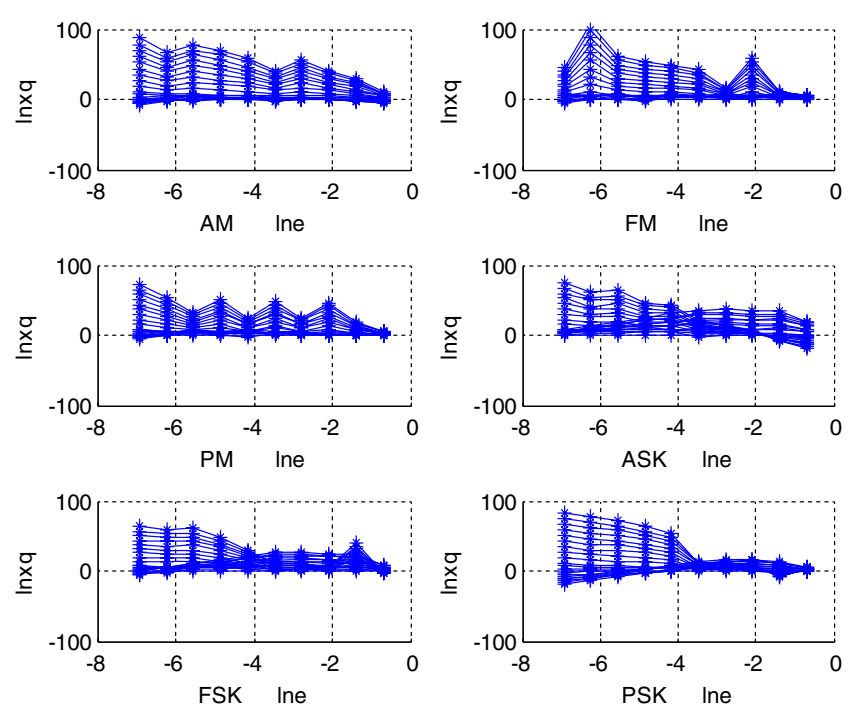

Fig. 7 Multi-fractal dimension-based features of different modulation signals under the SNR of $10 \mathrm{~dB}$.
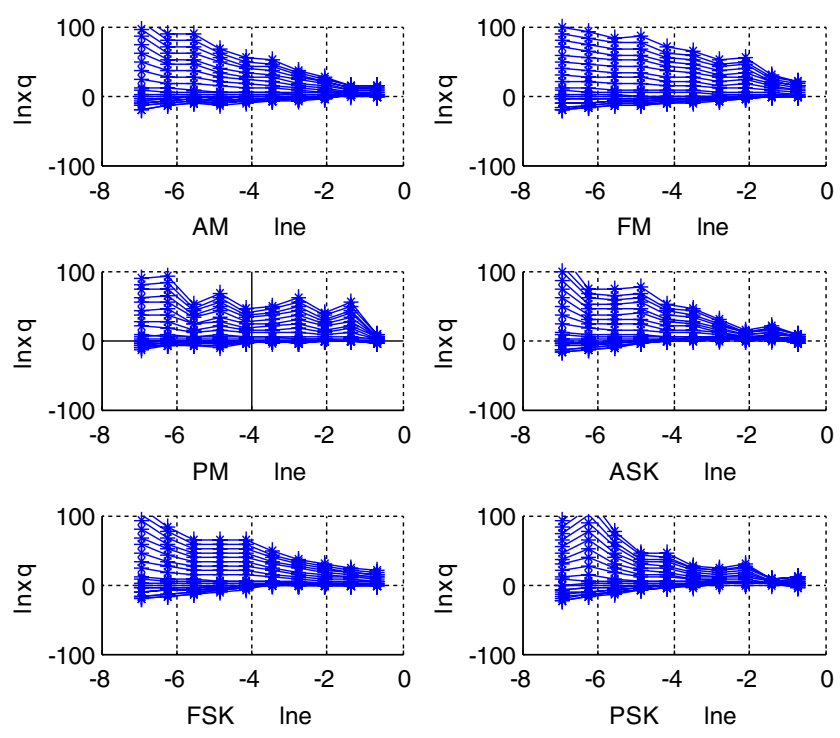

Fig. 8 Multi-fractal dimension-based features of different modulation signals under the SNR of $0 \mathrm{~dB}$.

number of the signals' points, and $y$-coordinate represents the amplitudes of signals. The figures on the right represents the multi-fractal dimensions of the signals. And while, $x$-coordinate Ine reprensents $\ln \varepsilon$, and $y$-coordinate Inxq represents $\ln X_{q}(\varepsilon)$ in Eq. (34).

From the simulation we can see that, fractal dimension curves of FSK signals with different distributed noise are different, so gray relation classifier can be used to calculate the correlation degree of each reconstructed sequence, which can achieve good recognition effect. A Monte Carlo simulation experiment is used to generate 1000 random 

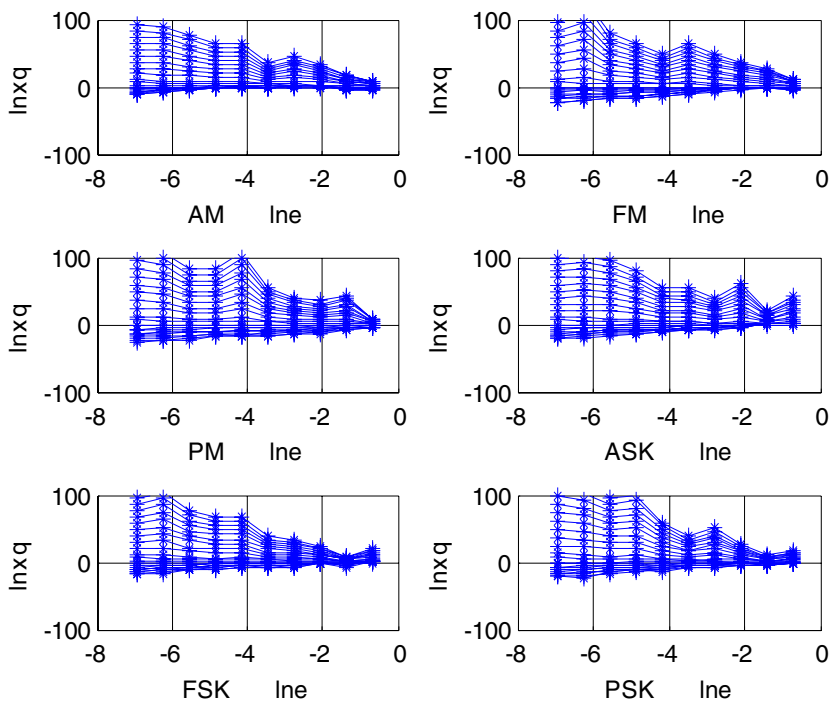

Fig. 9 Multi-fractal dimension-based features of different modulation signals under the SNR of $-10 \mathrm{~dB}$.

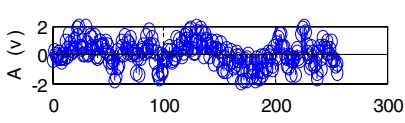

(a) $\mathrm{t}(\mathrm{ms})$

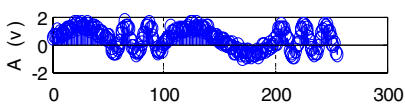

(b) $\mathrm{t}(\mathrm{ms})$
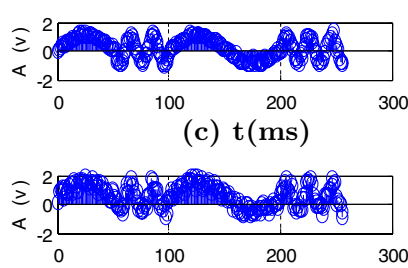

(d) $\mathrm{t}(\mathrm{ms})$

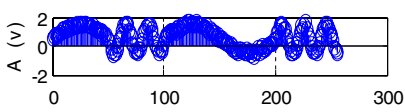

(e) $\mathrm{t}(\mathrm{ms})$ (c) $\mathbf{t}(\mathrm{ms})$

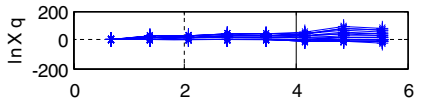

Ine

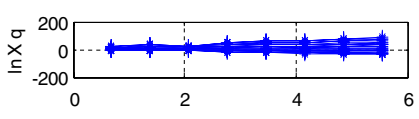

Ine

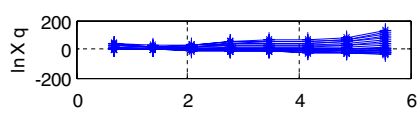

Ine

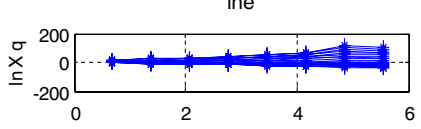

Ine

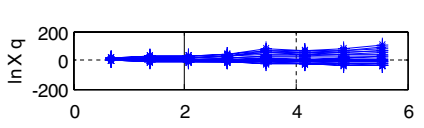

Ine
Fig. 10 (a) FSK signal + Uniform distributed noise, (b) FSK signal + Rayleigh distributed noise, (c) FSK signal + Exponential distributed noise, (d) FSK signal + Beta distributed noise, (e) FSK signal + Normal distributed noise, Multi-fractal dimension curves of FSK signals + different distribution noise sequences.

samples with different distribution to investigate the recognition effect of multi-fractal dimension of different distributed noise sequences. Simulation results are shown in Table 1

Information entropy is an important index to measure the uncertainty of signal's distribution and signal's complexity. It is often used to quantitatively describe the information contained in the signal, so
Table 1 Recognition Rate of Different Feature Extraction Methods Under Different SNR.

\begin{tabular}{lccccc}
\hline $\mathbf{S N R}(\mathbf{d B})$ & $\mathbf{- 2 0}$ & $\mathbf{- 1 0}$ & $\mathbf{0}$ & $\mathbf{1 0}$ & $\mathbf{2 0}$ \\
\hline $\begin{array}{c}\text { Recognition rate of } \\
\text { multi-fractal } \\
\text { dimension-based } \\
\text { features (\%) }\end{array}$ & 90.6 & 94 & 98 & 100 & 100 \\
$\begin{array}{l}\text { Recognition rate of } \\
\text { entropy-based } \\
\text { features }(\%)\end{array}$ & 80.8 & 83.2 & 85.4 & 94.6 & 97.6 \\
\hline
\end{tabular}

it also can be used to detect and extract the complexity of noise. Nowadays, information entropy has been widely used in many fields. Reference 23 used information entropy and probabilistic neural network together to identify the target in the sea battlefield, which receive good recognition result. In this experiment, the information entropy features and multi-fractal features of FSK signals with different distributed noise are extracted, respectively. The recognition results under different SNR environment are compared in Table 1, and the recognition time are shown in Table 2.

Simulation results show that multi-fractal curves of different distribution sequences are different, so gray relation theory can be used to distinguish them, which has a good recognition effect. From the comparison of Table 1, it can be seen that the recognition results of multi-fractal-based features of FSK signal with different distributed noise sequence is better than entropy-based feature. Even the signal has been completely submerged by noise at low SNR, it still has a relatively high recognition rate. However, comparing the recognition time, the entropy based feature extraction algorithm is slightly better than the multi-fractal-based feature extraction algorithm, that is, in the calculation complexity, the entropy-based algorithm is simpler than the multi-fractal-based algorithm.

Table 2 Simulation Time of Different Feature Extraction Methods.

\begin{tabular}{lc}
\hline $\begin{array}{l}\text { Recognition } \\
\text { Methods }\end{array}$ & $\begin{array}{c}\text { Time of Recognition } \\
\text { Processes (ms) }\end{array}$ \\
\hline $\begin{array}{l}\text { Multi-fractal-based feature } \\
\text { extraction method }\end{array}$ & 14.2 \\
$\begin{array}{l}\text { Entropy-based feature extraction } \\
\text { method }\end{array}$ & 10.2 \\
\hline
\end{tabular}




\section{CONCLUSIONS}

Fractal dimension is an important tool to describe the complexity of signals. Aiming at the problem that the signals' subtle features are difficult to extract in low SNR environment, this paper proposes a new feature extraction algorithm based on multi-fractal dimension and one-dimensional fractal dimension algorithm according to the complexity of the signal. For the signals with different noise, it is difficult to perform efficient feature extraction algorithm to get the subtle features in low SNR. Therefore, a multi-fractal dimension feature extraction algorithm based on phase space reconstruction is proposed. FSK signals with different distributed noise were used as the signals to be identified to verify the effectiveness of proposed algorithm. Simulation results show that, although the feature extraction algorithm based on multi-fractal dimension has a higher computational complexity than the one-dimensional feature extraction algorithm, it has better performance on the subtle features' extraction. Even in the SNR of $-10 \mathrm{~dB}$, it still has a high recognition rate.

\section{ACKNOWLEDGMENTS}

This work was supported by the National Nature Science Foundation of China (61301095 and 61603239), the Nature Science Foundation of Heilongjiang Province of China (F201408), the Key Development Program of Basic Research of China (JCKY2013604B001).

This paper is funded by the International Exchange Program of Harbin Engineering University for Innovation-oriented Talents Cultivation.

We gratefully thank the reviewers for their very useful discussions.

\section{REFERENCES}

1. Fu R.-R. Compound jamming signal recognition based on neural networks, in 2016 Sixth International Conference on Instrumentation and Measurement, Computer, Communication and Control (IMCCC) (2016), pp. 737-740.

2. S. Liu, et al. Numeric characteristics of generalized M-set with its asymptote, Appl. Math. Comput. 243 (2014) 767-774.

3. A. V. Makarenko, Deep learning algorithms for signal recognition in long perimeter monitoring distributed fiber optic sensors, 2016 IEEE 26th in Int. Workshop on Machine Learning for Signal Processing (MLSP) (2016), pp. 1-6.
4. H. Shi, J. Xiong, C. Zhou and S. Yang, A new recognition and classification algorithm of underwater acoustic signals based on multi-domain features combination, in 2016 IEEE/OES China Ocean Acoustics (COA) (2016), pp. 1-7.

5. X. Liu, R. Li, C. Zhao and P. Wang, Robust signal recognition algorithm based on machine learning in heterogeneous networks, J. Syst. Eng. Electron. 27(2) (2016) 333-342.

6. Y. Zhao, et al. Recognition of digital modulation signals based on high-order cumulants, in 2015 International Conference on Wireless Communications and Signal Processing (WCSP) (2015), pp. 1-5.

7. S. Liu et al. Fractal property of generalized $M$-set with rational number exponent, Appl. Math. Comput. 220 (2013) 668-675.

8. J. Li, A novel recognition algorithm based on holder coefficient theory and interval gray relation classifier, and KSII Trans. Internet Inform. Syst. (TIIS) 9(11) (2015) 4573-4584.

9. M. V. Gualsaquí Miranda, I. P. Vizcaíno Espinosa and M. J. Flores Calero, ECG signal features extraction, in 2016 IEEE Ecuador Technical Chapters Meeting (ETCM) (2016), pp. 1-6.

10. X. Kang, R. Tao and F. Zhang, Multiple-parameter discrete fractional transform and its applications, in IEEE Trans. Signal Process. 64(13) (2016) 34023417.

11. X. Yan et al. Innovative robust modulation classification using graph-based cyclic-spectrum analysis, IEEE Commun. Lett. 21(1) (2017) 16-19.

12. F. A. S. Borges, R. A. S. Fernandes, I. N. Silva and C. B. S. Silva, Feature extraction and power quality disturbances classification using smart meters signals, IEEE Trans. Ind. Inform. 12(2) (2016) 824833.

13. Q. Guo et al., Recognition of radar emitter signals based on SVD and AF main ridge slice, J. Commun. Netw. 17(5) (2015) 491-498.

14. A. H. Abdelaziz, S. Zeiler and D. Kolossa, Learning dynamic stream weights for coupled-HMM-based audio-visual speech recognition, IEEE/ACM Trans. Audio Speech Lang. Process. 23(5) (2015) 863-876.

15. X.-B. Qin, Y.-Z. Zhang, M.-T. Huang and M. Wang, EEG signal recognition based on wavelet transform and neural network, in 2016 International Symposium on Computer, Consumer and Control (IS3C) (2016), pp. 523-526.

16. S. Liu, et al., Distribution of primary additional errors in fractal encoding method, Multimed. Tools Appl. 76(4) (2017) 5787-5802.

17. A. Jiang and J. Gao, Fractal analysis of complex power load variations through adaptive multiscale filtering, in 2016 International Conference on Behavioral, Economic and Socio-cultural Computing (BESC) (2016), pp. 1-5. 
18. S. Liu, Z. Pan, W. Fu and X. Cheng, Fractal generation method based on asymptote family of generalized Mandelbrot set and its application, $J$. Nonlinear Sci. Appl. 10(3) (2017) 1148-1161.

19. P. Steffi Vanthana and A. Muthukumar, Iris authentication using gray level co-occurrence matrix and hausdorff dimension, in 2015 International Conference on Computer Communication and Informatics (ICCCI) (2015), pp. 1-5.

20. Y. Gui, Hausdorff dimension spectrum of self-affine carpets indexed by nonlinear fibre-coding, in 2009 International Workshop on Chaos-Fractals Theories and Applications (2009), pp. 382-386.
21. J. Li, and Y. Ying, Individual radiation source identification based onfractal box dimension, in The 2014 2nd International Conference on Systems and Informatics (ICSAI 2014) (2014), pp. 676-681.

22. A. A. Hajiannezhad and S. Mozaffari, Fractal and multi-fractal dimensions for farsi/arabic font type and size recognition, in 2011 7th Iranian Conference on Machine Vision and Image Processing (2011), p. 1.

23. N. Xiaobo, Z. Hu and C. Xinlai, Target recognition in naval battlefield based on information entropy and PNN, Electron. Opt. Control 17(4) (2010), 8386 . 